\title{
Post-Traumatic Stress Disorder in Dogonahawa and Zawan, North-Central Nigeria, Four-Years after Communal Violence: Prevalence and Risk Factors Prevalence of and risk factors for PTSD
}

\author{
Authors \\ Maigari Yusufu Taru, Moses David Audu, Davou Francis John, \\ Tungchama Friday Philip, Suwa Goden. Goar \\ Jos University Teaching Hospital (JUTH), Jos, Nigeria \\ Corresponding Author \\ Maigari Y Taru \\ Department of Psychiatry, Jos University Teaching Hospital, Plateau state Nigeria \\ Email: Tmaigariyusufu@yahoo.com, Tel: 2348036327951
}

\section{ABSTRACT}

Survivors of mass violence are at high risk of developing psychological disorders. However, little is known about the impact of trauma in post conflict low income countries. This study assessed the prevalence of and socio-demographic risk factors for posttraumatic stress disorder among conflict survivors in North-Central Nigeria in comparison to a control group drawn from a population that was not exposed to armed-conflict. This is a cross-sectional study that employed a multi stage sampling technique to select respondents in both groups. The Mini international neuropsychiatric interview was used to assess for symptoms of posttraumatic stress disorder and their socio-demographic variables obtained with socio-demographic questionnaire. The results showed that $55.5 \%$ and $9.8 \%$ of the respondents in the conflict exposed group and the control were diagnosed with posttraumatic stress disorder. Respondents exposed to mass violence were significantly more likely to experience posttraumatic stress disorder compared to the control group ( $p<0.001$ ). Being a female was the consistent risk factor for the disorder in both groups, while being never married and having individual monthly income below N20,000.00 were also predictive of posttraumatic stress disorder in the group exposed to conflict. In conclusion, posttraumatic stress disorder prevalence was higher among the group exposure to armedconflict. Thus, an effective model for immediate and long-term mental health support for trauma victims needs to be developed in order to reduce the incidence of posttraumatic stress disorder among conflict survivors.

Keywords: PTSD, sociodemographic predictors, mass violence, north-central Nigeria.

\section{INTRODUCTION}

Nigeria has witnessed recurrent political and ethno religious conflicts since the exit of the military in 1999; with the North Central region which Plateau State belongs to being one of the areas worst hit. For instance, Dogonahawa, a specific community in Jos South and part of
Barkin Ladi Local Government Areas (LGAs) of Plateau state was subjected to a severe armed attacked on March $7^{\text {th }}$ 2010. The attackers according to reports were allegedly unknown, close to 500 people mostly women and children were butchered with machetes, clubs, axes and some shot with guns in a surprised midnight raid 
that lasted over three hours, left several others injured, with houses and property destroyed and some survivors rendered homeless. Journalists broke down in tears as they saw hundreds of dead bodies inside houses, some strewn around the streets and pathways leading in and out of the village and others with head severed ${ }^{[1]}$.

Posttraumatic stress disorder (PTSD) is frequently reported among survivors who experienced an exceptionally stressful event of this nature. This disorder develops after a traumatic event that invoke actual or threatened death or serious injury or threat to the physical integrity of self or others and the individual initially responds with intense fear, helplessness or horror. The person later develops a response to the event characterized by persistent re-experiencing of the event, symptoms of numbness, avoidance and hyper arousal with consequent clinically significant distress or functional impairment ${ }^{[2]}$.

Previous studies across countries have reported a wide range of PTSD prevalence in general population, ranging from 12-month prevalence of $1.1 \% \%$ in Australia ${ }^{[3]}$ to lifetime prevalence of $7.8 \%$ in United States of America (USA) ${ }^{[4]}$, while a current PTSD prevalence of $9.4 \%$ was documented in Israeli ${ }^{[5]}$ general population. On the other hand, the results of studies conducted among post-conflict populations in Turkey ${ }^{[6]}$, Northern Ireland ${ }^{[7]}$, Lebanon ${ }^{[8]}$, Liberia ${ }^{[9]}$, Northern Uganda ${ }^{[10]}$, Rwanda ${ }^{[11]}$, and indeed Nigeria ${ }^{[12-14]}$ have demonstrated a variously high prevalence of Post-traumatic stress disorder in the range of $10-60 \%$. Correlates of PTSD found in these studies include gender, marital status, and income level.

These studies are however few in rural areas of low income countries including Nigeria that has continued to endure a variety of unresolved political, ethnic and religious conflicts amidst under-services in terms of security, social and health care.

It is against this backdrop that this study aims to conduct a community-based prevalence survey of
PTSD 4 years after the $7^{\text {th }}$ March 2010 communal attack in Dogonahawa (exposed group) in comparison to a control group drawn from Zawan (not-exposed group), a community similar in geographical location and cultural identity to Dogonahawa, but spared of the recurrent conflict affecting some communities in Plateau State for over a decade now and to examine the contributing factors associated with PTSD in these populations using a cross sectional study design

The study also hypothesized that PTSD will be significantly common among adults exposed to mass violence than the general population.

\section{METHOD}

This study was conducted 4years after the $7^{\text {th }}$ march 2010 communal violence in Dogonahawa. The control group was drawn from Zawan, a community with similar geographical location and cultural identity to Dogonahawa, but spared of the recurrent conflict affecting some communities in Plateau State for over a decade now. These are specific rural communities in Jos-South and part of Barkin Ladi Areas (LGAs) of Plateau State, with an estimated projected population of over 5500 people in Dogonahawa and 5300 in Zawan respectively (Projected with the 1991 national census) ${ }^{[15]}$. These people are predominantly the Berom ethnic group and most of them are farmers. The minimum sample size was calculated using Kish formula ${ }^{[16]}$ for cross sectional studies based on a prior estimate of $42 \%$ for PTSD ${ }^{[13]}$. Sample sizes of 260 and 250 were estimated for the exposed and not exposed groups respectively.

The study employed a multistage sampling of households to select respondents in both groups. Adults aged 18 years and above, resident in Dogonahawa and Zawan prior to and during the conflict were eligible for inclusion. Eligible adults with florid symptoms of mental illnesses and debilitating medical or surgical conditions which would impair their ability to participate in the study were excluded. 
Data was collected by the researcher and the research assistants who are fluent in both English and Hausa languages.

The instruments were administered to the participants on an individual basis in their respective homes, in a face-to-face interview ensuring that the participants had enough privacy.

The socio-demographic questionnaire was administered first and thereafter the Mini International Neuropsychiatric Interview (M.I.N.I) [17] PTSD module was used to assess current symptoms of PTSD. The M.I.N.I was designed as a brief structured interview for the major Axis I psychiatric disorders in DSM-IV and ICD-10.It specifically asks question about the past month symptoms of PTSD. The PTSD module of this instrument has been used in a similar study carried out in Nigeria ${ }^{[12]}$.

In this study, the stem (trauma screen) question of the MINI was modified and adapted to reflect exposure to the March $7^{\text {th }}, 2010$ communal attack as: "about 4 years ago, this Community was subjected to severe armed attack in which many people were close to, but escaped death or witnessed the murder of a relative, friend, neighbor or stranger, or sexual violence involving self or others or property burnt, destroyed or lost. Participants were asked not to describe the nature of the trauma they experienced in order to avoid the risk that people would under-report, because of a wish to avoid memory of or gain psychological distance from the event. The control respondents were also asked not to state or describe the nature of the trauma they experienced but if they so wish, they could describe it.

Individual whose symptoms fulfilled the DSM$1 \mathrm{~V}$ criteria was assigned a diagnosis of PTSD.

The Hausa translated version of the questionnaire was used to collect data from respondents with no formal education.

Ethical approval was obtained from the ethical committee of the Jos University Teaching Hospital, while permission was granted by the Ward Heads of Dogonahawa and Zawan respectively. Written informed consent was obtained from individual study subjects.

\section{Data Analysis}

The statistical package for social sciences version 16 (SPSS-16) Software package was used to analyze the data. The results were presented in frequency tables, means, standard deviation and descriptive analysis. T-test was used to compare mean values of numerical variables and chi-square test was used to investigate the difference between categorical variables and their associations. Values of $\mathrm{P}<0.05$ were considered statistically significant. To predict the risk for

PTSD, the significant independent variables were then entered into a logistic regression analysis.

\section{RESULTS}

\section{Socio-demographic characteristics of respondents at the time of the study}

A total of 510 respondents were interviewed, but 499 interviews comprising of 254(97.7\%) from the conflict exposed group and $245(98 \%)$ from the not-exposed group were used in the analyses. The remaining interviews were excluded because of incomplete data.

The age range of respondents in the exposed group was between 18 to 82 years and their mean age was $34.6 \pm 13.6$. The not-exposed group was older, with an age range between 18 to 84 years and their mean age was $40.7 \pm 17.8$ years. The group not exposed were significantly older than the exposed group $(\mathrm{t}=<0.001)$

Both groups were similar in gender distribution, with slightly more males than females represented in the exposed; 137(53.9\%) males and notexposed; 131(53.5\%) males.

The widowed respondents were significantly more likely to be represented in the exposed group than the not exposed $(\mathrm{P}<.001)$. The sample however comprised of a high proportion of married respondents in both the exposed group $132(52.0 \%)$ and not-exposed group 171(69.8\%). 
Though the predominant ethnic group in both samples was Berom, the two groups however differed significantly, with the other ethnic minorities being more represented in the notexposed group than the exposed group, $(\mathrm{P}<0.001)$. In the exposed group, the sample comprised of respondents that were significantly less likely to have someone available to talk to after the trauma $(\mathrm{P}=<0.001)$ and to feel safe living in the community after the trauma $(\mathrm{P}=<0.001)$ than the not-exposed group. See table1.

\section{Prevalence of PTSD}

Among the 254 respondents interviewed in the exposed group using M.I.N.I, 141(55.5\%) of them were diagnosed as having current PTSD, while $24(9.8 \%)$ of the 245 respondents in the not exposed group were diagnosed as having current PTSD.

The prevalence of PTSD among the two groups was found to differ at a level that was statistically significant, with adults in the exposed group being more likely to have PTSD than the group not exposed $(\mathrm{P}<0.001)$. See table2.

\section{Sociodemographic correlates of respondents with and without PTSD}

In the conflict exposed group, there were significantly more females, $80(68.4 \%)$ than males, $61(44.5 \%)$ with diagnosis of PTSD $(\mathrm{P}<0.001)$. Similarly, respondents with PTSD were significantly more likely to be never married, $(\mathrm{P}=0.006)$ and had individual monthly income below $<20,000.00, \quad(\mathrm{P}<0.001)$. In the same manner, respondents who had no one available to talk to immediately after the trauma were significantly more likely to have PTSD than those with someone available to talk to $(\mathrm{P}=0.019)$. So also are those feeling unsafe living in the community after the trauma being significantly more likely to have PTSD than those feeling safe living in the community after the trauma, $(\mathrm{P}=0.001)$. See table 3 .
Among the group not-exposed, only being a female rather than a male and being previously married compare to being married, were significantly associated with PTSD $(\mathrm{p}=0.003)$ and $(\mathrm{P}<0.001)$ respectively. See table4.

\section{Predictors of PTSD among the exposed group}

Among the exposed group, females were three times more likely to have PTSD than males, $(\mathrm{P}=0.001)$. Having individual monthly income below 20,000.00 was >8 times more likely to have PTSD compared to individual monthly income of $\geq 50,000.00 \quad(\mathrm{P}=0.003)$. Other variables were not found to predict PTSD at statistically significant levels. See table5.

Among the group not exposed, the odds of having PTSD was 4 times more likely in females than males $(\mathrm{P}=0.008)$. Though being previously married tripled the odds of having PTSD compared to being a widow, but was not at statistically significant levels $(\mathrm{P}=0.274)$. See table6. 
Table1: Characteristics of respondents in the conflict exposed and the control groups

\begin{tabular}{|c|c|c|c|c|c|c|c|}
\hline \multicolumn{8}{|c|}{ Frequency (\%) } \\
\hline Variable & Response & $\begin{array}{l}\text { Conflict victims } \\
\text { group }(n=254)\end{array}$ & $\begin{array}{l}\text { Controls } \\
(\mathrm{n}=245)\end{array}$ & $\begin{array}{c}\text { Total } \\
(\mathrm{N}=499)\end{array}$ & \multicolumn{3}{|c|}{$\begin{array}{c}\text { Statistics } \\
\text { df }\end{array}$} \\
\hline Age group(years) & 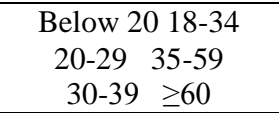 & $\begin{array}{l}123(48.4) \\
93(36.6 \%) \\
38(15.0 \%)\end{array}$ & $\begin{array}{l}83(33.9) \\
94(38.4) \\
68(27.7)\end{array}$ & $\begin{array}{l}206(41,3) \\
187(37.5) \\
106(21.2)\end{array}$ & 16.630 & 2 & $<0.001$ \\
\hline & Mean \pm SD & $34.33 \pm 13.6$ & $40.49 \pm 17.5$ & $37.35 \pm 10$ & \multicolumn{3}{|c|}{$\mathrm{t}=4.378, \quad \mathrm{P}<0.001$} \\
\hline Gender & $\begin{array}{l}\text { Male } \\
\text { Female }\end{array}$ & $\begin{array}{l}137(51.1) \\
117(50.6)\end{array}$ & $\begin{array}{l}131(48.9) \\
114(49.4)\end{array}$ & $\begin{array}{l}268(100.0) \\
231(100.0)\end{array}$ & 0.011 & 1 & 0.917 \\
\hline Education & $\begin{array}{l}\text { No formal education } \\
\text { Primary education } \\
\text { Secondary education } \\
\text { Tertiary education } \\
\end{array}$ & $\begin{array}{c}41(16.1) \\
86(33.9) \\
104(40.9) \\
23(9.1) \\
\end{array}$ & $\begin{array}{l}55(22.5) \\
90(36.7) \\
75(30.6) \\
25(10.2) \\
\end{array}$ & $\begin{array}{c}96(19.2) \\
176(35.3) \\
179(35.9) \\
48(9.6) \\
\end{array}$ & 6.754 & 3 & 0.080 \\
\hline Marital status & $\begin{array}{c}\text { Married } \\
\text { Never married } \\
\text { Previously married } \\
\text { Widowed }\end{array}$ & $\begin{array}{c}132(52.0) \\
76(29.9) \\
14(5.5) \\
32(12.6)\end{array}$ & $\begin{array}{c}171(69.8) \\
49(20.0) \\
17(6.9) \\
8(3.3)\end{array}$ & $\begin{array}{c}303(60.7) \\
125(25.1) \\
31(6.2) \\
40(8.0)\end{array}$ & 25.38 & 3 & $<0.001$ \\
\hline Occupation & $\begin{array}{c}\text { Professionals } \\
\text { Non - professionals } \\
\text { Unemployed }\end{array}$ & $\begin{array}{c}16(6.3) \\
176(69.3) \\
62(24.4)\end{array}$ & $\begin{array}{c}10(4.1) \\
181(73.9) \\
54(22.0)\end{array}$ & $\begin{array}{c}26(5.2) \\
357(71.6) \\
116(23.2)\end{array}$ & 1.845 & 2 & 0.398 \\
\hline Income & 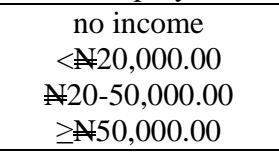 & $\begin{array}{c}46(18.1) \\
131(51.6) \\
59(23.2) \\
18(7.1) \\
\end{array}$ & $\begin{array}{c}38(15.5) \\
118(48.2) \\
77(31.4) \\
12(4.9) \\
\end{array}$ & $\begin{array}{c}84(16.8) \\
249(49.9) \\
136(27.3) \\
30(6.0) \\
\end{array}$ & 4.862 & 3 & 0.182 \\
\hline Ethnicity & $\begin{array}{l}\text { Berom } \\
\text { Others }\end{array}$ & $\begin{array}{c}250(53.3) \\
4(13.8)\end{array}$ & $\begin{array}{c}220(46.7) \\
25(86.2)\end{array}$ & $\begin{array}{c}470(100.0) \\
29(100.0)\end{array}$ & 16.96 & 1 & $<0.001$ \\
\hline Religion & $\begin{array}{l}\text { Christianity } \\
\text { Muslim }\end{array}$ & $\begin{array}{c}253(99.6) \\
1(0.4 \%)\end{array}$ & $\begin{array}{c}242(98.8) \\
3(0.2)\end{array}$ & $\begin{array}{c}495 \\
3(100.0)\end{array}$ & & & \\
\hline Trauma exposure & $\begin{array}{l}\text { Yes } \\
\text { No }\end{array}$ & $\begin{array}{c}254(100.0) \\
0(0.0)\end{array}$ & $\begin{array}{c}60(18.9) \\
185(19.2)\end{array}$ & $\begin{array}{l}314(100.0) \\
185(100.0)\end{array}$ & - & - & - \\
\hline Someone to talk to & $\begin{array}{l}\text { Yes } \\
\text { No }\end{array}$ & $\begin{array}{c}22(40.7) \\
232(89.2)\end{array}$ & $\begin{array}{l}32(59.3 \%) \\
28(10.8 \%)\end{array}$ & $\begin{array}{c}54(100.0) \\
260(100.0)\end{array}$ & & & $<0.001$ \\
\hline Sense of safety & $\begin{array}{l}\text { Yes } \\
\text { No }\end{array}$ & $\begin{array}{c}38(46.9) \\
216(92.7) \\
\end{array}$ & $\begin{array}{c}43(53.1) \\
17(7.3) \\
\end{array}$ & $\begin{array}{c}81(100.0) \\
233(100.0)\end{array}$ & 81.53 & 1 & $<0.001$ \\
\hline PTSD prevalence & $\begin{array}{l}\text { Yes } \\
\text { No }\end{array}$ & $\begin{array}{l}141(55.5) \\
113(44.5)\end{array}$ & $\begin{array}{c}24(9.8) \\
221(90.2)\end{array}$ & $\begin{array}{l}165(33.1) \\
334(66.9)\end{array}$ & 117.762 & 1 & $<0.007$ \\
\hline
\end{tabular}

Table 2: Prevalence and distribution of PTSD

\begin{tabular}{|l|c|c|c|}
\hline Posttraumatic stress disorder & Exposed group & Not-group & Total \\
\hline Yes & $141(55.5 \%)$ & $24(9.8)$ & $165(33.1 \%)$ \\
\hline No & $113(44.5 \%)$ & $221(90.2 \%)$ & $334(76.9 \%)$ \\
\hline Total & $254(100 \%)$ & $245(100 \%)$ & $499(100 \%)$ \\
\hline
\end{tabular}

Table 3: Exposed group with and without PTSD

\begin{tabular}{|c|c|c|c|c|c|c|c|}
\hline \multicolumn{8}{|c|}{ Frequency $=\mathrm{N}(\%)$} \\
\hline Variables & Response & $\begin{array}{c}\text { Yes } \\
n=141\end{array}$ & $\begin{array}{c}\text { No } \\
n=113\end{array}$ & Total $\mathrm{N}=254$ & Statistics & & \\
\hline Age group(years) & $\begin{array}{c}18-34 \\
35-59 \\
>60\end{array}$ & $\begin{array}{l}69(48.9) \\
54(38.3) \\
18(12.8)\end{array}$ & $\begin{array}{l}\text { 54(47.8) } \\
39(34.5) \\
19(17.7)\end{array}$ & $\begin{array}{c}123(48.4) \\
93(36.6) \\
38(15.0)\end{array}$ & 1.263 & 2 & 0.527 \\
\hline Gender & $\begin{array}{c}\text { Male } \\
\text { Female }\end{array}$ & $\begin{array}{l}61(44.5) \\
80(68.4)\end{array}$ & $\begin{array}{l}76(55.5) \\
37(31.6)\end{array}$ & $\begin{array}{l}137(100.0) \\
117(100.0)\end{array}$ & 14.536 & 1 & $<0.001$ \\
\hline Level of education & $\begin{array}{l}\text { No formal educat } \\
\text { primary } \\
\text { secondary } \\
\text { tertiary }\end{array}$ & $\begin{array}{c}24(17.0) \\
52(36.9) \\
56(39.7) \\
9(6.9)\end{array}$ & $\begin{array}{l}17(15.0) \\
34(30.1) \\
48(42.5) \\
14(12.4)\end{array}$ & $\begin{array}{c}41(16.1) \\
86(33.9) \\
104(40.9) \\
23(9.1)\end{array}$ & 3.622 & 3 & 0.305 \\
\hline Marital status & $\begin{array}{c}\text { Married } \\
\text { Never married } \\
\text { Previously married } \\
\text { Widowed }\end{array}$ & $\begin{array}{c}60(42.6) \\
54(38.3) \\
9(6.4) \\
18(12.8)\end{array}$ & $\begin{array}{c}72(63.7) \\
22(19.5) \\
5(4.4) \\
14(12.4)\end{array}$ & $\begin{array}{c}132(52.0) \\
76(29.9) \\
14(5.5) \\
32(12.6)\end{array}$ & 13.282 & 3 & 0.004 \\
\hline Occupation & $\begin{array}{c}\text { Professional } \\
\text { Non-professionals }\end{array}$ & $\begin{array}{c}7(14.3) \\
34(79.2)\end{array}$ & $\begin{array}{c}1(0.9) \\
11(9.5)\end{array}$ & $\begin{array}{c}2(1.2) \\
17(10.3)\end{array}$ & 15.623 & 2 & 0.065 \\
\hline
\end{tabular}


JMSCR Vol||05||Issue||02||Pages 17231-17240||February

\begin{tabular}{|c|c|c|c|c|c|c|c|}
\hline & Unemployed & $8(16.3)$ & $34(29.3)$ & $42(25.5)$ & & & \\
\hline Income level & $\begin{array}{c}\text { No income } \\
<\$ 20,000.00 \\
\$ 20-50,000.0 \\
\geq \$ 50,000.00\end{array}$ & $\begin{array}{c}25(17.7) \\
89(63.1) \\
23(16.3) \\
4(2.8)\end{array}$ & $\begin{array}{l}21(18.6) \\
42(37.2) \\
36(31.9) \\
14(12.4) \\
\end{array}$ & $\begin{array}{c}46(18.1) \\
131(51.6) \\
59(23.2) \\
18(7.1) \\
\end{array}$ & 22.821 & 3 & $<0.001$ \\
\hline Religion & $\begin{array}{l}\text { Christianity } \\
\text { Islam }\end{array}$ & $\begin{array}{c}141(55.7) \\
0(0.0)\end{array}$ & $\begin{array}{c}112(44.3) \\
1(100.0)\end{array}$ & $\begin{array}{c}253(100.0) \\
1(100.0) \\
\end{array}$ & 0.012 & 1 & 0.912 \\
\hline Ethnicity & $\begin{array}{l}\text { Berom } \\
\text { Others }\end{array}$ & $\begin{array}{c}139(98.6) \\
2(50.0)\end{array}$ & $\begin{array}{l}111(1.4) \\
2(50.0) \\
\end{array}$ & $\begin{array}{c}250(100.0) \\
4(100.0)\end{array}$ & 0.050 & 1 & 0.823 \\
\hline Trauma exposure & $\begin{array}{l}\text { Yes } \\
\text { No }\end{array}$ & $\begin{array}{c}141(85.5) \\
0(0.0)\end{array}$ & $\begin{array}{c}113 \\
0(0.0)\end{array}$ & $\begin{array}{c}254(100.0) \\
0(0.0)\end{array}$ & - & - & - \\
\hline Someone available & $\begin{array}{l}\text { Yes } \\
\text { No } \\
\end{array}$ & $\begin{array}{c}7(31.8) \\
134(57.8) \\
\end{array}$ & $\begin{array}{l}15(68.2) \\
98(42.2) \\
\end{array}$ & $\begin{array}{c}22(100.0) \\
232(100.0) \\
\end{array}$ & 5.475 & 1 & 0.019 \\
\hline Sense of safety & $\begin{array}{l}\text { Yes } \\
\text { No }\end{array}$ & $\begin{array}{c}12(31.6) \\
129(59.7)\end{array}$ & $\begin{array}{l}26(68.4) \\
87(40.3)\end{array}$ & $\begin{array}{c}38(100.0) \\
216(100.0)\end{array}$ & 10.364 & 1 & $<0.001$ \\
\hline
\end{tabular}

Table 4: Control group with and without PTSD

\begin{tabular}{|c|c|c|c|c|c|c|c|}
\hline \multicolumn{8}{|l|}{ Frequency=N(\%) } \\
\hline Variables & Response & Yes $n=24$ & $\begin{array}{c}\text { No } \\
n=221\end{array}$ & Total $\mathrm{N}=245$ & \multicolumn{3}{|c|}{ Statistic } \\
\hline Age group (years) & $\begin{array}{c}18-34 \\
35-59 \\
\geq 60 \\
\end{array}$ & $\begin{array}{c}9(37.5) \\
12(50.0) \\
3(12.5)\end{array}$ & $\begin{array}{l}74(33.5) \\
82(37.1) \\
65(29.4)\end{array}$ & $\begin{array}{l}83(33.9) \\
94(38.4) \\
68(27.7) \\
\end{array}$ & .3 .272 & 2 & 0.195 \\
\hline \multicolumn{8}{|c|}{$1.11 \pm 17.95, \quad 40.49 \pm 17.52$} \\
\hline Gender & $\begin{array}{c}\text { Male } \\
\text { Female }\end{array}$ & $\begin{array}{c}6(4.5) \\
18(15.9)\end{array}$ & $\begin{array}{c}126(95.5) \\
95(84.1)\end{array}$ & $\begin{array}{l}132(100.0) \\
113(100.0)\end{array}$ & 8.929 & 1 & 0.003 \\
\hline Level of education & $\begin{array}{l}\text { Noformal educat } \\
\text { primary } \\
\text { secondary educat } \\
\text { tertiary }\end{array}$ & $\begin{array}{c}6(25.0) \\
10(41.7) \\
5(20.8) \\
3(12.5) \\
\end{array}$ & $\begin{array}{l}49(22.2) \\
80(36.2) \\
70(31.7) \\
22(9.9)\end{array}$ & $\begin{array}{l}55(22.5) \\
90(36.7) \\
75(30.6) \\
25(10.2) \\
\end{array}$ & 1.222 & 3 & 0.748 \\
\hline Marital status & $\begin{array}{c}\text { Married } \\
\text { Never married } \\
\text { Previously married } \\
\text { Widowed }\end{array}$ & $\begin{array}{c}10(41.7) \\
5(20.8) \\
7(29.2) \\
2(8.3)\end{array}$ & $\begin{array}{c}161(73.3) \\
44(19.9) \\
10(4.1) \\
6(2.7)\end{array}$ & $\begin{array}{c}171(70.2) \\
49(20.0) \\
17(6.5) \\
8(3.3)\end{array}$ & 16.570 & 3 & 0.001 \\
\hline Occupational group & $\begin{array}{c}\text { Professional } \\
\text { Non-professional } \\
\text { Unemployed }\end{array}$ & $\begin{array}{c}2(8.3) \\
18(75.0) \\
4(16.7)\end{array}$ & $\begin{array}{c}8(3.6) \\
163(73.8) \\
50(22.6)\end{array}$ & $\begin{array}{c}10(4.1) \\
181(73.9) \\
54(22.0)\end{array}$ & 1.316 & 1 & 0.518 \\
\hline Individual income/month & $\begin{array}{c}\text { No income } \\
<\$ 20,000.00 \\
\$ 20-50,000.0 \\
>\$ 50,000.00 \\
\end{array}$ & $\begin{array}{c}4(16.7) \\
15(62.5) \\
3(12.5) \\
2(8.3) \\
\end{array}$ & $\begin{array}{c}34(15.4) \\
103(46.6) \\
74(33.5) \\
10(4.5) \\
\end{array}$ & $\begin{array}{c}38(15.5) \\
118(48.2) \\
77(31.4) \\
12(4.9) \\
\end{array}$ & 4.833 & 3 & 0.184 \\
\hline Religion & $\begin{array}{c}\text { Christianity } \\
\text { Islam }\end{array}$ & $\begin{array}{c}24(9.9) \\
0(0.0) \\
\end{array}$ & $\begin{array}{c}218(90.1) \\
3(100.0) \\
\end{array}$ & $\begin{array}{c}242(100.0) \\
3(100.0)\end{array}$ & - & - & - \\
\hline Ethnicity & $\begin{array}{l}\text { Berom } \\
\text { Others }\end{array}$ & $\begin{array}{l}20(9.1) \\
4(15.4)\end{array}$ & $\begin{array}{l}199(90.9) \\
22(84.6)\end{array}$ & $\begin{array}{l}219(100.0) \\
26(100.0)\end{array}$ & 0.442 & 1 & 0.506 \\
\hline $\begin{array}{l}\text { Previous history of trauma } \\
\qquad(\mathrm{n}=60)\end{array}$ & $\begin{array}{l}\text { Yes } \\
\text { No }\end{array}$ & $\begin{array}{l}2(20.0) \\
22(44.0)\end{array}$ & $\begin{array}{l}8(80.0) \\
28(56.0)\end{array}$ & $\begin{array}{l}10(100.0) \\
50(100.0)\end{array}$ & 1.125 & 1 & 0.289 \\
\hline $\begin{array}{l}\text { Someone available to talk to } \\
\qquad(\mathrm{n}=60)\end{array}$ & $\begin{array}{l}\text { Yes } \\
\text { No }\end{array}$ & $\begin{array}{l}10(31.2) \\
14(50.0)\end{array}$ & $\begin{array}{l}22(68.8) \\
14(50.0)\end{array}$ & $\begin{array}{l}32(100.0) \\
28(100.0)\end{array}$ & 2.188 & 1 & 0.139 \\
\hline $\begin{array}{l}\text { Sense of safety } \\
(n=60)\end{array}$ & $\begin{array}{l}\text { Yes } \\
\text { No }\end{array}$ & $\begin{array}{l}19(44.2) \\
5(29.4)\end{array}$ & $\begin{array}{l}24(55.8) \\
12(70.6)\end{array}$ & $\begin{array}{l}43(100.0) \\
17(100.0)\end{array}$ & 1.108 & 1 & 0.293 \\
\hline
\end{tabular}


Table 5: Factors associated with PTSD among the exposed group.(Dogonahawa)

\begin{tabular}{|l|c|c|c|c|}
\hline Variables & Odd ratio & \multicolumn{2}{|c|}{$95 \%$ Confidence interval } & \\
\hline IGender & 1.000 & Lower & Upper & P \\
Male & 2.868 & 1.592 & 5.167 & $<0.001$ \\
\hline Female & 0.602 & 0.255 & 1.417 & 0.245 \\
\hline Marital status & 2.935 & 1.111 & 7.754 & 0.030 \\
Married & 2.750 & 0.614 & 12.320 & 0.186 \\
Never married & 1.000 & & & \\
Previously married & 2.438 & 0.551 & 10.797 & 0.240 \\
Widowed & 8.698 & 2.137 & 35.407 & 0.003 \\
Individual monthly income & 3.274 & 0.755 & 14.199 & 0.113 \\
Non income & 1.000 & & & \\
$<20,000.00$ & 0.831 & 0.215 & 3.217 & 0.789 \\
N20,000.00-N50,000.00 & 1.000 & & & \\
$\geq$ N50,000.00 & 0.397 & 0.143 & 1.103 & 0.076 \\
\hline Someone available to talk to & 1.000 & & & \\
Yes & & & \\
No & & & \\
Sense of safety & & & \\
Feeling Safe & & & \\
Not feeling &
\end{tabular}

Table 6: Factors associated with PTSD among the not-exposed group (Zawan)

\begin{tabular}{|l|c|c|c|c|}
\hline & & \multicolumn{2}{|c|}{ 95\%Confidence interval } & \\
\hline Variables & Odd ratio & Lower & Upper & P \\
\hline Sex & 3.920 & 1.422 & 10.804 & 0.008 \\
Male & 1.000 & & & \\
Female & & & & \\
\hline Marital status & 0.255 & 0.044 & 1.486 & 0.129 \\
Married & 0.456 & 0.069 & 3.023 & 0.416 \\
Never married & 2.963 & 0.423 & 20.744 & 0.274 \\
Previously married & 1.000 & & & \\
Widowed &
\end{tabular}

\section{DISCUSSION}

This study found that, four years after exposure to armed attack in Dogonahawa (exposed group), the proportion of adult_respondents meeting the DSM$1 \mathrm{~V}$ diagnostic criteria for PTSD was 55.5\%, whereas $9.8 \%$ was found in Zawan (not exposed group). This result is expected for the reason that all the participants in Dogonahawa (exposedgroup) were screened positive for traumatic experiences related to the $7^{\text {th }}$ march armed attack, compared to one-quarter of the participants in Zawan (not exposed group) that were screened positive for trauma exposure. Thus, a greater number of respondents in Dogonahawa were more likely to meet diagnostic criteria for PTSD, which includes witnessing a traumatic event as a necessary condition. This supports our hypothesis that PTSD will be significantly common among adults exposed to mass violence than the general population.
Our findings are consistent with previous reports of PTSD prevalence in Nigeria by Beiser and colleagues ${ }^{[14]}$, and Akinyemi etal ${ }^{[12]}$, who recorded PTSD prevalence of $60 \%$ and $37 \%$ among conflict victims and $14.5 \%$ and $13.7 \%$ among their controls drawn from the general peaceful population. In particular, the prevalence of PTSD found among the post conflict survivors (55.5\%) was similar to the $24.8-54 \%$ current prevalence found among post conflict survivors in Rwanda ${ }^{[1]}$, Lebanon ${ }^{[8]}$, Liberia ${ }^{[9]}$ and Uganda [10], but higher than the $10-15.1 \%$ found in Northern-Ireland ${ }^{[6]}$, and Turkey ${ }^{[7]}$, following exposure to mass violence in these countries.

Similarly, the rate in the group not exposed to conflict was close to the $9.4 \%$ prevalence of current PTSD reported among the Israeli general population ${ }^{[5]}$.

The observed disparity in prevalence of PTSD in the present study, as well as previous findings in 
other low income countries vis-a-vis the Surveys in Turkey ${ }^{[6]}$ and Northern Ireland ${ }^{[7]}$ is not surprising. Perhaps, most trauma survivors in low income countries continued to be re-traumatized due to absence of basic needs like food, clothing, shelter, not to mention daily post trauma stressors like financial difficulties, infectious diseases and high mortality. However, the difficulty in comparing studies of different populations exposed to armed-conflict has to be emphasized because of variability in factors such as level of trauma exposure; time elapsed between exposure and diagnosis, other methodological differences and cultural factors.

This study revealed that, being a female rather than a male was a common significant predictor for PTSD in both groups, which is in consonant with the findings of other previous studies that reported same ${ }^{[4,8,10]}$. In support of this, it has been documented that women use dissociative defense mechanism more than men and also have more negative self-schemas and world-schemas than men following exposure to traumatic events. These factors have been found to be consistent with increased risk of PTSD ${ }^{[18]}$.

We found that respondents that were never married among the exposed group and previously married in the group not-exposed were more likely to develop PTSD than those married. Creamer, P. Burgess and McFarlane ${ }^{[3]}$ and Kessler et al $^{[4]}$ in their respective studies found that; previously married men and women are at greater risk of developing PTSD than those currently married. Being previously married/never married may be associated with reduced level of social support, independence and self-esteem, with consequent weakening of the person's defense against trauma-induced stressor thereby increasing individual's risk of developing psychological disorders ${ }^{[19]}$. This suggests that marriage itself confers some social support which serves as a buffer against developing PTSD.

Among the trauma victims, having low individual monthly income and in particular, having estimated individual monthly income below $\$ 20$, 000.00 was found to be one of the strongest demographic predictors for PTSD. Studies in Northern Ireland ${ }^{[6]}$ and Lebanon ${ }^{[8]}$ revealed that the coincidence of violence and poverty increased the risk of PTSD. This is presumed to be the same in Nigeria which is a low income country with majority of its population living in rural areas and indeed, below poverty line. Living in low-income environment also places an individual at increased risk of PTSD because of financial constraints which deprives them of the basic necessities of life, like food, shelter, clean water and health care services. In addition, they lack the resources to rebuild their lives after communal violence.

In this study, a high proportion of respondents in the exposed group reported having no one available to talk to immediately after the trauma and are still worried about their safety living in the community four years after the armed conflict. While these factors may be linked to the onset of PTSD, the former may not explain the persistence of high rate of current PTSD at four years after the trauma because of the social support network which includes extended family ties, the Churches and neighbors in the communities. This form of living arrangement provides the potential for consistent social support, which in turn is a key resilient factor in promoting emotional wound healing. On the other hand, the awareness of ongoing attacks on some communities in Plateau State may be one of the salient perpetuating factors for PTSD symptoms among the conflict victims. The explanation could be that they continue to feel insecure, which can be associated with persistent anxiety levels and the expectation that another armed-attack could happen. Dejong et al ${ }^{[20]}$ revealed that the relatively high prevalence of PTSD, 34\% in Algeria compared to other areas under that study may be due to the fact that terrorist attacks were still ongoing during the time of their data collection.

Other socio-demographic variables such as ethnicity, religion, and occupation were not 
significantly associated with PTSD in both groups as against previous studies that have demonstrated this association ${ }^{[6,9]}$. Worthy of note is the fact that the samples were drawn from populations not displaced by the conflict or other traumatic events in the case of the control group and therefore had their social structure and cultural institutions preserved. Thus, most of their traditional life was not disrupted to an extent to which it can be significantly associated with PTSD.

The study however had limitations that also need to be acknowledged. First the current findings are specific to the two rural communities in Plateau State, North-Central Nigeria and not necessarily applicable to other parts of Nigeria, particularly urban areas. Secondly, we focused on PTSD, while depression, alcohol use disorders and other anxiety disorders may be part of the response to trauma in some respondents. Furthermore, this study assumed that the findings were related to the 7th March 2010 armed attack on Dogonahawa, rather than other past traumatic exposures. It is possible that PTSD diagnosed with some respondents may have occurred as a result of traumatic events experienced prior to or after the 2010 armed attack on this community.

The strength of this study lies on the fact that it is the first comparative community-based survey to examine the Prevalence of PTSD after exposure to armed conflict in the North-Central region of Nigeria and the fact that the predictors were consistent with previous research, supports the validity of the findings.

In conclusion, this study developed measures to assess the prevalence of current PTSD, as well determined the predictors of PTSD among adult population exposed to armed conflict and those not exposed. The main finding from this study was that the prevalence of PTSD was significantly higher among adult population exposed to mass violence compared to the group that was not exposed $(\mathrm{P}=<0.001)$. Being a female was a common predictor of PTSD in both groups, while being never married and having individual monthly income below N20,000.00 were also predictive of PTSD in the group exposed to mass violence.

The high rate of PTSD, unavailability of people to talk to, coupled with the feeling of insecurity by majority of the respondents is a cause for serious concern because social support, safety and feeling of hope for the future are crucial for developing resilience which is necessary for healing and recovery. Hence, there is need for trauma healing programs for such traumatized people.

\section{ACKNOWLEDGEMENT}

The authors wish to thank the Resident Doctors and Psychologists of the Jos University Teaching Hospital (JUTH) who assisted with data collection. We would also like to thank the community leaders and the respondents in Dogonahawa and Zawan for their cooperation during the survey.

\section{Sources of support in the form of grants-Nil}

\section{REFERENCES}

1. Daily champion Nigeria: The most recent violence in Plateau State posted on $8^{\text {th }}$ March 2010.

2. American Psychiatric Association. Diagnostic and Statistical Manual of Mental Disorders (DSM-IV). 1994; 4th Edition .Washington, D.C.

3. Creamer M, Burgess P, McFarlane AC. Posttraumatic stress disorder: Findings from Australian National Survey of mental health and wellbeing. Psychological Medicine 2001; 31: 1237-1247.

4. Kessler RC, Sonnega A, Bromet E, Nelson CB: Posttraumatic stress disorder in National Comorbidity Survey. Arch Gen Psychiatry 1995, 52:1048-1060.

5. Bleich A, Gelkopf M, Solomon Z. Exposure to terrorism, stress-related mental health symptoms, and coping behaviors among a nationally representative sample in Israel. Journal of 
the American Medical Association. JAMA. 2003;290(5):612-620. doi:10.1001/jama.290.5.612

6. Aziz Yasan, Günay Saka, Meliksah Ertem, , Mustafa Ozkan, \& Mehmet Ataman. Prevalence of PTSD and related factors in communities living in conflictual area: Diyarbakir case:Torture 2008, Volume 18.

7. Orla T. Muldoon, Ciara Downes Social identification and post-traumatic stress symptoms in post-conflict Northern Ireland.The British Journal of Psychiatry Jul 2007, 191 (2) 146-149; DOI: 10.1192/bjp.bp.106.022038

8. Farhood LF, Noureddine SN. PTSD, depression, and health status in Lebanese civilians exposed to a church explosion. Int J Psychiatry Med. 2003;33(1):39-53.

9. Galea S, Rockers PC, Saydee G, Macauley R, Varpilah ST, Kruk ME. Persistent psychopathology in the wake of civil war: long-term posttraumatic stress disorder in Nimba County, Liberia http://www.ncbi.nlm.nih.gov/pubmed/206 344612010;100 (9):1745-51.

10. Bayard Roberts, Kaducu felix Ocaka, John Browne, Thomas Oyok and Eybert Sondol factors associated with post-traumatic stress disorder and Depression amongst internally displaced person in Northern Uganda. BMC Psychiatry,2008, 8:38.

11. Pham PN, Harvey M, Timothy L. Trauma and posttraumatic stress disorder symptoms in Rwanda. Journal of American Medical Association JAMA. 2004 Aug 4;292(5):602-12.

12. Obilom RE, Thatcher TD. Posttraumatic Stress Disorder following ethno religious conflict in Jos. Nigeria. Journal of Interpersonal Violence 2008 Aug; 23 (8):1108-19.

doi: $10.1177 / 0886260507313975$
13. Oluwaseun O Akinyemi, Eme T Owoaje, Olusimbo K Ige and Oluwafemi A Popoola. A comparative study of mental health and quality of life in long term refugees and host populations in OruIjebu, Southwest Nigeria: BMC ResearchN otes 2012,5:394. DOI: 10.1186/1756-0500-5-394

14. Beiser M, Wiwa O, Adebajo S. Human initiated disaster, social disorganization and posttraumatic stress disorder above Nigeria's oil basins. Social Sciences and Medicine 2010; 71(2): 221-227. doi: 10.1016/j.socscimed.2010.03.039

15. National Bureau of Statistic. Federal Republic of Nigeria 2006, Population Census. Official Gazatte(FGP) 71/52007/2,500 (PL24): Legal notice on publication of the details of the breakdown of the National and State Provisional Census 2006

16. Kish L. Survey Sampling. John Whilley and Sons, New York 1965

17. Sheehan DV, Lecruibier Y, HarnettSheehan K, The Mini International Neuropsychiatric Interview. J Clin Psychiatry 1998; 59(20):22-33.

18. Breslau N, Kessler R, Chilcoat H, Davis G, Andreski P: Trauma and posttraumatic stress disorder in the community: the 1996 Detroit area survey of trauma. Arch Gen Psychiatry 1998, 55:626-632

19. Marcia A Voges and David M Romney. Risk and resiliency factors in posttraumatic stress disorder: Annals of General Hospital Psychiatry 2003, 2:4

20. de Jong J, Komproe IH, Ommeren MV, Masri ME, Araya M, Khaled $\mathrm{N}$ et al. Lifetime events and posttraumatic stress disorder in 4 post conflict settings. The Journal of the American Medical Association 2001; 286: 555-562. 